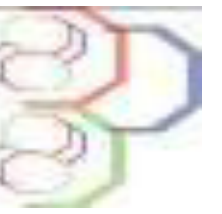

Journal of Applied Biosciences 107: 10381-10394

ISSN 1997-5902

\title{
Restauration des écosystèmes dégradés de l'Aire Marine Protégée de Saint-Louis par l'immersion de récifs artificiels et le reboisement de la mangrove : impacts sur la biodiversité marine et côtière (Saint-Louis, Sénégal).
}

Serigne Modou SARR ${ }^{*}$, Mouhameth CAMARA ${ }^{1}$, Amsatou THIAM $^{1}$, Momy SECK $^{1}$ et Mignane SARR ${ }^{2}$ 1 Institut Supérieur de Formation Agricole et Rurale (ISFAR) ex ENCR, Université de Thiès, BP 54 Bambey, Sénégal 2 Aire Marine Protégée de Saint-Louis, Ministère de l'Environnement et du Développement Durable, Sénégal

*Auteur Correspondant ; E-mail : sarmodou@yahoo.fr; Tél. (+221) 774358017

Original submitted in on $5^{\text {th }}$ October 2016. Published online at www.m.elewa.org on $30^{\text {th }}$ November 2016 http://dx.doi.org/10.4314/jab.v107i1.4

\section{RESUME}

Objectif: L'objectif de cette étude est d'évaluer la contribution de l'immersion de récifs artificiels et du reboisement de la mangrove sur la restauration des écosystèmes dégradés au niveau de l'Aire Marine Protégée (AMP) de Saint-Louis et des zones périphériques.

Méthodologie et résultats : Pour déterminer les impacts de l'immersion des récifs artificiels et du reboisement de la mangrove, nous avons procédé à une analyse comparative des rapports de l'état de référence de 2009 et de la pêche expérimentale de 2015, ainsi que des enquêtes auprès des pêcheurs de la commune de SaintLouis, avec un échantillon de 97 unités de production (un pêcheur par unité de production) et auprès des femmes transformatrices des huîtres de la commune de Ndiébène Gandiol (village de Diél Mbam), avec aussi un échantillon de 37 femmes, ainsi que des entretiens avec les gestionnaires et partenaires de l'AMP. Cette évaluation a aussi fait l'objet d'une expérience d'évaluation de la productivité de la mangrove en termes de ressources malacologiques. Les résultats obtenus ont permis de montrer que l'immersion de récifs est jugée non satisfaisante par les pêcheurs interrogés, contrairement au reboisement de la mangrove considéré comme satisfaisant par les femmes transformatrices des huîtres et les gestionnaires de l'AMP. Une évolution des taxons et du poids qui passe respectivement de 25 à 34 taxons et de 73 à $87,223 \mathrm{~kg}$ est notée lors de la pêche de 2009 et celle de 2015. Une productivité de la mangrove de six (6) potamides et de treize (13) de crabes violonistes en moyenne est également notée par $\mathrm{m}^{2}$.

Conclusion et application : En somme, cette étude nous a permis de montrer que l'immersion de récifs artificiels et le reboisement de la mangrove ont des impacts relativement positifs sur la biodiversité marine et côtière. Les résultats obtenus à partir de cette méthode ont montré que l'étude peut être utilisée comme modèle d'évalution pour des écosystèmes exploités.

Mots clés : Restauration, écosystème, récifs artificiels, mangrove, AMP, Saint-Louis 

marine et côtière (Saint-Louis, Sénégal).

Restoration of degraded ecosystems of the Marine Protected Area of Saint- Louis by the immersion of artificial reefs and mangrove reforestation: impacts on marine and coastal biodiversity (Saint-Louis, Senegal).

\begin{abstract}
Objective: The objective of this study is to evaluate the contribution of the immersion of artificial reefs and mangrove reforestation on degraded ecosystem restoration at the Marine Protected Area (MPA) of St. Louis and surrounding areas.

Methodology and Results: To determine the effects of immersion and artificial reforestation of mangrove reefs, we conducted a comparative analysis of reports from the baseline of 2009 and the experimental fishery in 2015, as well as creel surveys of the town of Saint-Louis, with a sample of 97 production units (a fisherman per unit of production) and among women processors of oysters of the common Ndiébène Gandiol (village Diel Mbam), with also -a sample of 37 women, as well as interviews with MPA managers and partners. This assessment has also been an evaluation experience productivity of mangroves in terms of molluscan resources. The results have shown that immersion reefs are considered unsatisfactory by the fishermen interviewed, unlike the mangrove reforestation considered satisfactory by women processors of oysters and MPA managers. An evolution of taxa and weight passes respectively 25 and 34 taxa and 73 to $87.223 \mathrm{~kg}$ is noted when fishing in 2009 and that of 2015. Productivity mangrove six (6) Potamides and thirteen (13) burrows of fiddler crabs on average is also noted by $\mathrm{m}^{2}$.

Conclusion and Application of result: In sum, this study allowed us to show that immersion of artificial reefs and mangrove reforestation has relatively positive impacts on marine and coastal biodiversity. The results obtained from this method have shown that the study can be used as a model for Evaluation of exploited ecosystems Keywords: Restoration, ecosystem, artificial reefs, mangroves, AMP, Saint-Louis
\end{abstract}

\section{INTRODUCTION}

L'économie sénégalaise a pendant longtemps, reposé sur l'arachide et les phosphates. Avec les années successives de sécheresse et la détérioration des termes de l'échange suite au choc pétrolier, la pêche est devenue très vite le premier secteur économique (CRODT, 2000). La pêche occupe une place prépondérante dans les politiques publiques de création d'emplois particulièrement le sous-secteur artisanal. Elle représente une source de revenus et une activité primordiale au sein même de la région de Saint-Louis (ANSD 2015). Cependant, suite à l'augmentation de la demande en poissons aussi bien à l'intérieur qu'à l'extérieur du pays, une intensification des activités de la pêche industrielle et artisanale est observée. Cette activité est menacée pour un ensemble de raisons que sont: l'augmentation de l'effort de pêche, des prélèvements directs ou accidentels de certaines espèces, la dégradation de la mangrove et les pollutions provoquées par les activités humaines sur le littoral. Une insuffisance des mesures de conservation et le non-respect des lois et règlements pour la pérennisation des ressources halieutiques sont constatés et ainsi que les changements climatiques. La pêche illicite, non déclarée et non réglementée (INN) a également appauvri les stocks de poissons, détruit les habitats marins, entraîné une distorsion de concurrence pour les pêcheurs honnêtes et affaibli les communautés côtières, notamment dans les pays en voie de développement (Commission Européenne, 2015). Dudley \& al. (2000) rapportent que I'Industrie Pétrolière et les déversements détruisent les habitats et portent atteinte à la biodiversité, endommagent des forêts de mangrove, des récifs coralliens et des pêcheries, à la suite d'accidents graves et de fuites régulières. Force est de reconnaître que ces activités reposent sur un espace sensible qui montre parfois des signes de dégradation inquiétants: destruction d'habitats naturels, pollutions, érosion, salinisation des sols, Niang (2010). Cette menace est à l'origine de nombreux impacts négatifs sur les écosystèmes 


\section{Sarr et al., J. Appl. Biosci. 2016. Restauration des écosystèmes dégradés de l'Aire Marine Protégée de Saint-Louis par l'immersion de récifs artificiels et le reboisement de la mangrove : impacts sur la biodiversité marine et côtière (Saint-Louis, Sénégal).}

marins et côtiers qui occasionnent plusieurs dommages dont : la surexploitation des ressources halieutiques, la dégradation des fonds marins et la pollution des eaux aux plans chimiques (accidents de transport de carburant, polluant domestique et industriel) et physique (perte d'engins continuant à pêcher, usage d'explosifs et de substances toxiques). La communauté internationale réunie à Durban en 2003 a recommandé la création d'aires marines protégées (AMP) pour le renforcement des mesures de conservation des ressources halieutiques. Par décret 2004-14-08 du 04 Novembre 2004, le Sénégal a créé cinq (5) AMP dont celle de Saint-Louis. Cette dernière doit répondre à la fois aux objectifs de conservation de la biodiversité marine et côtière et au développement socio-économique sans pour autant nuire aux

\section{MATERIEL ET METHODES}

La zone d'étude: L'Aire Marine Protégée (AMP) de Saint-Louis est située dans le département de Saint-Louis, sur la façade maritime de la Commune de Ndiébèn Gandiol et la commune de Saint-Louis, sur la Langue de Barbarie entre l'ancienne embouchure du ressources halieutiques. L'ouverture de la brèche aux années 2003 pour solutionner le trop plein d'eau a pour conséquences la dégradation de la mangrove et des sites de nidification des oiseaux. L'effort de pêche considérable au niveau de la région de SaintLouis (3847 pirogues et plus de 22.000 pêcheurs) et l'ouverture de la brèche ont des conséquences néfastes sur les ressources halieutiques. Cependant, dans le cadre des activités d'aménagement de l'AMP de Saint-Louis, un programme de restauration des écosystèmes marins et côtiers est mis en place pour contribuer à l'atteinte des objectifs de conservation du site. C'est ainsi que nous essayons de déterminer les impacts que peuvent avoir ces activités de restauration des habitats dégradés sur la diversité halieutique et sur l'avifaune.

fleuve Sénégal et le quartier pêcheur de Guet-Ndar (figure 1). Elle a été créée par le Décret présidentiel $n^{\circ}$ 2004-1408 du 04 novembre 2004 sur une aire de $496 \mathrm{~km}^{2}$ (49 600 ha). 

marine et côtière (Saint-Louis, Sénégal).

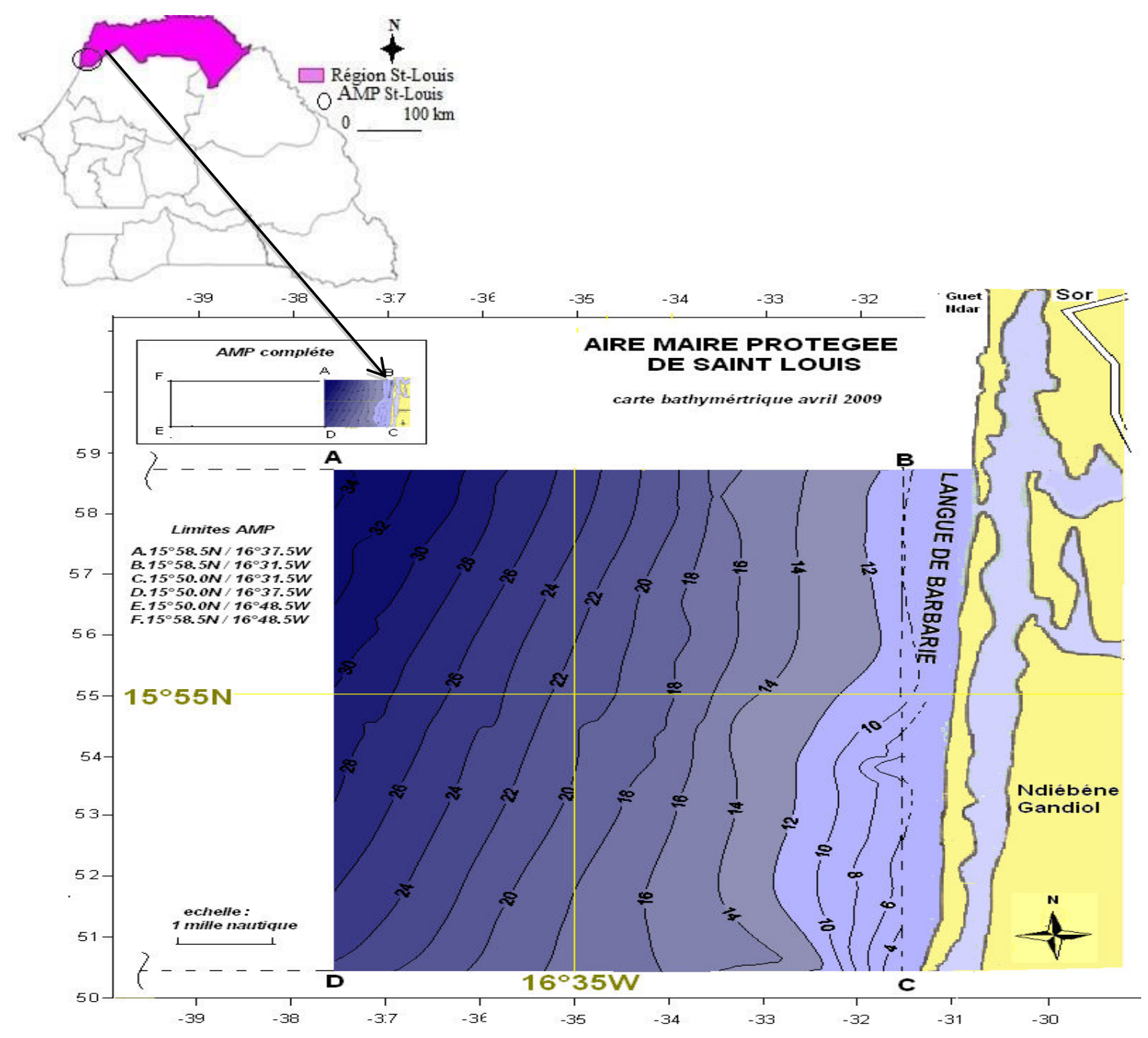

Figure 1 : Carte de localisation de la zone d'étude

Matériel : Pour réaliser cette présente étude, nous avons utilisé :

- $\quad$ un questionnaire administré aux pêcheurs de Saint-Louis pour recueillir auprès des pêcheurs des informations relatives aux causes de dégradations des écosystèmes marins et cotiers, les conséquences de la dégradation, les activités de restauration et les impacts des récifs artificiels sur les ressources halieutiques ;

- un questionnaire administré aux femmes transformatrices des huîtres à Diél Mbam qui a également permis de recueillir des informations sur l'impact du reboisement de la mangrove sur les produits malacologiques et sur l'avifaune ;

un guide d'entretien destiné aux gestionnaires et partenaires de l'AMP qui a permis de recueillir le point de vue des différents acteurs sur l'impact des activités de restauration des écosystèmes dégradés sur la biodiversité marine et côtière. Cette interrogation a permis de déduire des indicateurs de suivi de ces activités.

- $\quad$ une fiche de suivi de la mangrove reboisée en 2012 à Diél Mbam qui a également permis de mesurer les paramètres physico-chimiques de la zone reboisée, de dénombrer les ressources malacologiques et de déterminer les indices de présence d'oiseaux ; - des rapports de l'état de référence de 2009 et de la pêche expérimentale de 2015 ;

- des logiciels tels que Sphinx, Past, Quantum gis, Google earth et un progiciel Excel ;

- des appareils de mesure des paramètres physico-chimiques à savoir un pH-mètre, un salinomètre, un thermo-hygromètre, ainsi qu'un GPS garmin et un appareil photo de marque Sony 6 mega pixels. 

marine et côtière (Saint-Louis, Sénégal).

Méthodes : Échantillonnage : pour déterminer le nombre de pêcheurs et de femmes transformatrices interrogées, nous avons adopté la méthode de Fisher (Diop, 2006) qui fait intervenir trois paramètres ( $\mathrm{Ne}, \mathrm{N}$ et $\mathrm{n}$ ).
$\mathrm{Ne}=$ taille de l'échantillon à interroger

$\mathrm{N}=$ taille de la population estimée à 3847 unités de production

$n=1 / d^{2}$

$$
\begin{gathered}
N e=\frac{n}{\left[1+\left(\frac{n}{N}\right)\right]} \text { avec } d=\text { erreur de } 10 \% \text { et } n=\frac{1}{d^{2}} N e=\frac{\frac{1}{d^{2}}}{\left.\left.\frac{1}{d^{2}}\right)\right]} \\
\quad N e=\frac{\frac{1}{d^{2}}}{\left[1+\left(\frac{N}{d^{2}}\right)\right]}
\end{gathered}
$$

$\mathrm{Ne}=97$ unités de production dont un (1) pêcheur par unité de production, ce qui donne quatre-vingt-dix-sept (97) pêcheurs interrogés, pour la plupart il s'agit des capitaines de navigation qui sont généralement d'origine Saint-Louisienne, dans l'objectif d'obtenir des résultats fiables.

$\mathrm{N}=256$ femmes transformatrices

Et avec $d=15 \%$, nous avons trente-sept (37) femmes transformatrices interrogées.

Un guide d'entretien destiné aux gestionnaires de l'AMP est élaboré pour trianguler les informations. II regroupe des questions relatives aux différentes causes de la dégradation des écosystèmes, de leurs conséquences sur les ressources, des impacts du reboisement de la mangrove et des indicateurs de suivi de ces activités de restauration des écosystèmes dégradés. Ensuite, nous avons procédé à une expérience sur une parcelle de mangrove de $90 \mathrm{~m}^{2}$ reboisée en 2012 pour mesurer les paramètres physico-chimiques de la zone et déterminer la productivité de la mangrove en termes de ressources malacologiques. L'expérience consistait à définir neuf (9) placettes de $1 \mathrm{~m}^{2}$ chacune dans la parcelle de mangrove, puis nous avons déterminé les coordonnées géographiques de chaque placette et de la parcelle, dénombrer les ressources malacologiques et les indices de présence d'oiseaux.

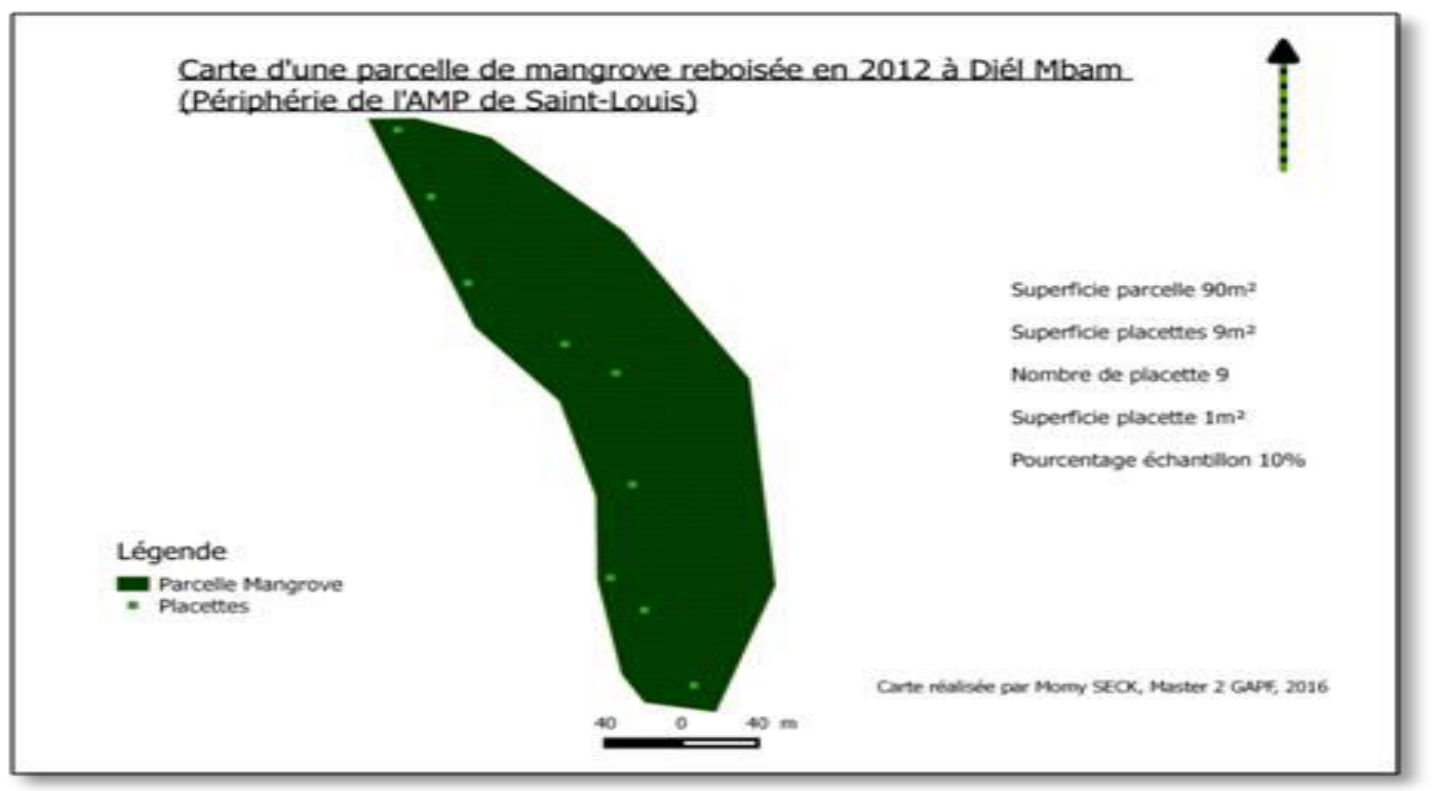

Figure 2 : Carte d'une parcelle de mangrove reboisée en 2012 à Diél Mbam (village périphérique de l'AMP de SaintLouis) 

marine et côtière (Saint-Louis, Sénégal).

Nous avons également effectué une analyse comparative des résultats de l'état de référence de 2009 et de la pêche expérimentale de 2015. Lors de l'état de référence de 2009, trois (3) stations de pêche ont été retenues et trois (3) coup de pêche ont eu lieu dans chaque station. Ces dernières se trouvent à Keur Bernard, Keur Barka et Pilote qui correspondent respectivement aux positions de début, de milieu et de fin de l'AMP. Ce choix est fait après concertation avec les pêcheurs et les membres des comités de gestion. Les pêches ont eu lieu le jour principalement à 9heures 30 et à 18heures, pour une durée moyenne de 45 minutes avec une senne de plage de $250 \mathrm{~m}$ de longueur avec une hauteur de chute de $4 \mathrm{~m}$. La maille est plus grande sur les ailles $(30 \mathrm{~mm})$ et plus petite au fond (10mm au niveau de la poche). En 2015, neuf (9) stations sont retenues, et dans chaque station un coup de pêche a été effectué. Ce choix est fait sur la

\section{RESULTATS}

Typologie des acteurs enquêtés : L'analyse de la figure 3 révèle que $51,55 \%$ des pêcheurs interrogés ne sont pas instruits en français mais en arabe, et seuls $48,45 \%$ ont un niveau d'étude différent (de la Classe d'Initiation à l'école élémentaire à la classe de terminale au moyen secondaire). Cela prouve que la scolarisation des

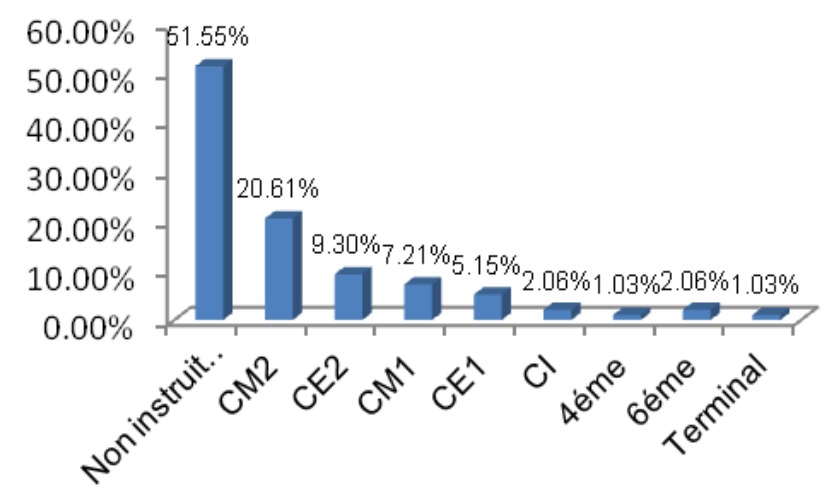

base d'un zonage de l'AMP qui indique les lieux autorisés pour la pêche, et la zone de protection intégrale et aussi la liste des espèces locales devant faire l'objet d'un suivi scientifique particulier après l'état référence de 2009. Cependant, six types d'engins de pêche sont utilisés notamment deux filets maillants dérivants de surface, une senne tournante, un filet dormant, un palangre, une senne de plage et un filet épervier suivant un calendrier prédéfini.

Traitement des données: les coordonnées géographiques sont traitées sur Excel puis exporté sur Quantum-gis pour la cartographie de la parcelle et la digitalisation des placettes. Les données des questionnaires sont traitées avec Sphinx et Excel, et celles des deux pêches expérimentales avec Past et Excel.

habitants de la commune de Saint-Louis (particulièrement Guet Ndar et Goxu Mbacc) est très influencée par la pêche. Donc la sensibilisation des enfants au niveau des écoles coraniques doit être encouragée pour une meilleure prise de conscience sur l'importance de la conservation des ressources halieutiques.

Figure 3 : scolarisation des pêcheurs enquêtés

En ce qui concerne l'exploitation des huîtres dans la mangrove, toutes les femmes transformatrices interrogées ne sont pas instruites en français mais en arabe.L'analyse de la figure 4 indique que les principales zones de pêche des enquêtés sont l'embouchure avec $51,10 \%$ et la Mauritanie $36,80 \%$, ensuite le fleuve avec $4,3 \%$ et les zones de Cayar, Dakar, Casamance, Mbour et la sous-région avec respectivement $2,4 \%, 1,8 \%, 0,6 \%$, $0,6 \%$ et $2,4 \%$. Cette forte concentration de pêcheurs dans l'embouchure qui correspond à l'entrée de l'AMP affecte négativement les zones de repos, de nurserie et de reproduction de la biodiversité marine par le bruit des moteurs des pirogues. 


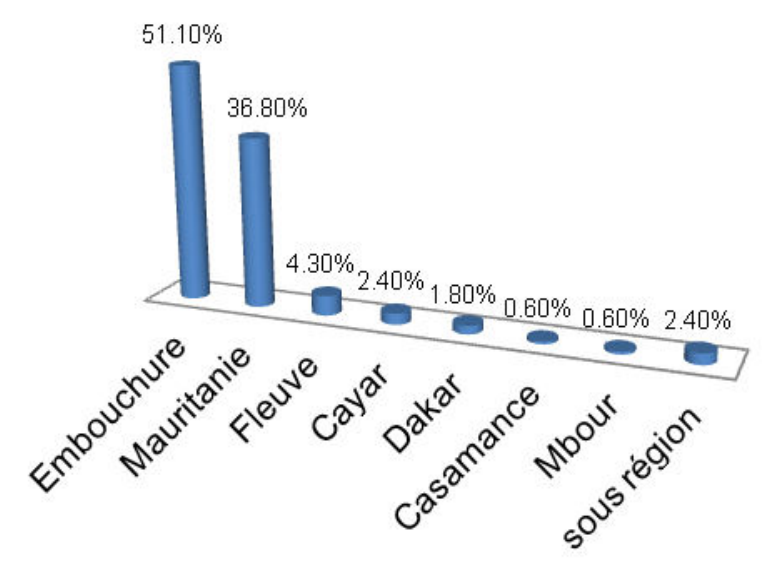

Figure 4 : différentes zones de pêche des enquêtés

Concernant les femmes, la figure 5 montre que leur principale zone de cueillette des huîtres est Diél Mbam selon $87,70 \%$ des enquêtées, ensuite les zones périphériques de l'AMP pour $11,2 \%$ et puis au niveau des guirlandes confectionnées avec l'aide d'un bailleur pour 1,10\%. L'exploitation de ces huîtres s'effectue

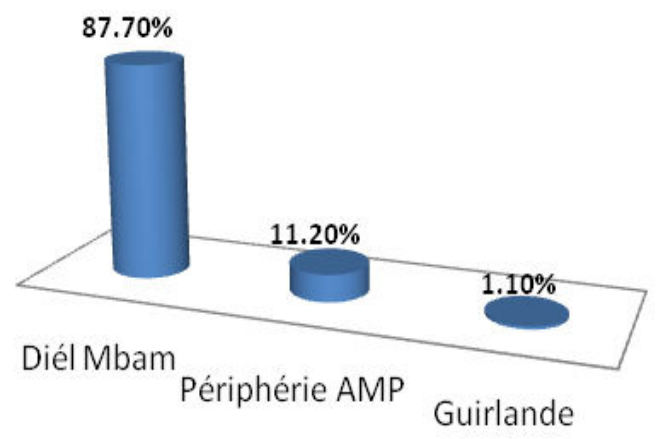

Figure 5 : principales zones de cueillette des huîtres

\section{Niveau d'information des acteurs}

- $\quad$ Niveau de connaissance de l'existence d'une zone de protection intégrale (ZPI) : L'étude de la figure 6 montre que $85 \%$ des enquêtés sont au courant de l'existence d'une zone de protection intégrale à l'opposé naturellement dans la mangrove (racines de palétuviers) et que seule 1,1\% utilise des guirlandes. La confection de ces dernières doit être encouragée pour une exploitation rationnelle des ressources malacologiques et une gestion durable des écosystèmes de mangrove. de $15 \%$ qui l'ignorent. Ce qui montre qu'une bonne partie des pêcheurs est informée par la visibilité des balises et les missions de sensibilisations effectuées dans cette zone.

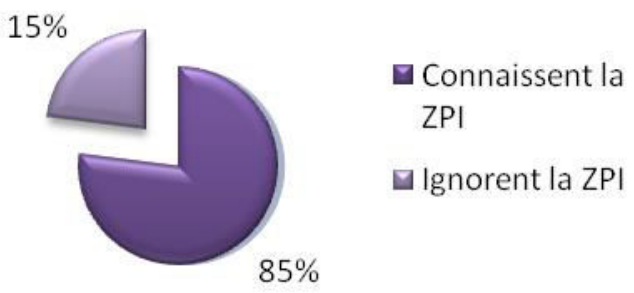

Figure 6 : Taux de connaissance de l'existence d'une Zone de Protection Intégrale (ZPI) 

Saint-Louis par l'immersion de récifs artificiels et le reboisement de la mangrove : impacts sur la biodiversité marine et côtière (Saint-Louis, Sénégal).

- $\quad$ Niveau de connaissance de l'immersion de récifs artificiels : L'analyse des résultats de la figure 7 montre que $77 \%$ des enquêtés étaient au courant de l'immersion de récifs artificiels contre $23 \%$ de notre échantillon. Donc une bonne partie des pêcheurs est informée des activités de restauration des habitats dégradés au niveau de l'AMP de Saint-Louis.

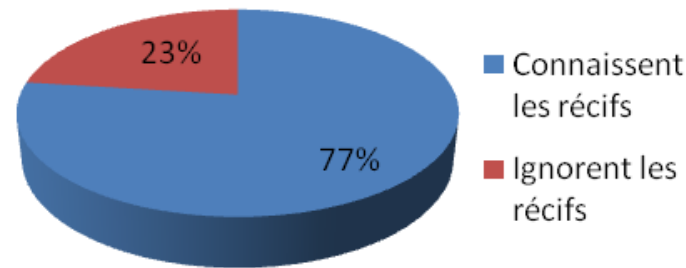

Figure 7 : Taux de connaissance de l'immersion de récifs artificiels

- $\quad$ Niveau de connaissance du reboisement de la mangrove : Toutes les femmes interrogées étaient au courant des activités de reboisement de la mangrove et ont eu à y participer durant les différentes années de reboisement. Leur forte mobilisation est due à l'importance accordée aux biens et services rendus par les écosystèmes de mangrove, comme source de revenus et ayant une fonction de protection et de fixation des sols. Un impact socio-économique positif envers la population à travers l'exploitation des ressources malacologiques est noté.

Les impacts des activités de restauration des écosystèmes dégradés : En 2009, I'AMP de Saint-Louis dans le cadre de ses activités d'aménagement, a bénéficié d'un appui de Compact/FEM, pour la confection et l'immersion de trois cent deux (302 récifs artificiels) dans les zones de frayères (Zone de Protection Intégrale) avec une technologie locale. Puis en 2014, sous le financement du projet FEM, l'Aire Marine Protégée de Saint-Louis à travers le GIE Suxali AMP a confectionné et immergé quatre cents (400) récifs artificiels pour contribuer à l'atteinte des objectifs de conservation du site. Pour ce qui est du reboisement de la mangrove vingt-cinq (25) hectares ont été reboisés en 2012 sous le financement du projet FEM. En 2015 également le même GIE a effectué un reboisement de mangrove sur une superficie de trente (30) hectares dans six sites au niveau de la zone périphérique de l'AMP de Saint-Louis sous le financement du projet GIZC. Cette activité s'inscrit dans le cadre des stratégies d'adaptation aux changements climatiques par la réhabilitation des écosystèmes de mangroves. Pour une bonne productivité de la mangrove en termes de ressources malacologiques, il faut une durée de cinq (5) ans car la mise en place d'une biodiversité nécessite un long processus.

- L'impact de l'immersion des récifs artificiels sur les ressources halieutiques

L'analyse comparative des résultats de l'état de référence de 2009 et de la pêche expérimentale de 2015 : montre une évolution du nombre de taxons qui passe de vingt-cinq (25) en 2009 à trente-quatre (34) en 2015. Cette évolution est également notée au niveau du poids qui passe aussi de $73 \mathrm{~kg}$ à $87,223 \mathrm{~kg}$ captures lors des deux pêches expérimentales (Figure 8). 

marine et côtière (Saint-Louis, Sénégal).

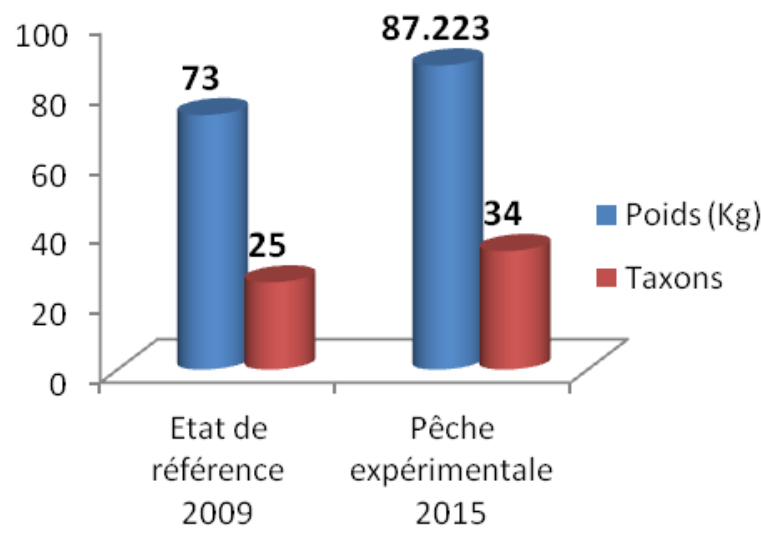

Figure 8 : Evolution des taxons et du poids des captures

Pour une période de six (6) ans, le nombre de taxons a évolué de neuf (9) points avec un différentiel de poids de $14,223 \mathrm{~kg}$. Seules 12 espèces que sont Brachydeuterus auritus, Chlorocombis chrysurus, Drepane africana, Cynoglossus senegalensis, Ephippion guttifer, llisha africana, Pentanemus quinquarius, Pseudotolithus senegalensis, Sardinella aurita, Sardinella maderensis, Stromateus fiatola et Trichiurus lepturus sont communes aux deux pêches. Ainsi, ces 12 espèces en commune peuvent être considérées comme étant la base permanente du peuplement de l'AMP de Saint-Louis en saison froide. Cependant, en 2015, environ 402 individus composés de 34 espèces, appartenant à 20 familles ont été identifiées. En termes de diversité spécifique la famille des Scianidea et des Mugilidae avec quatre (4) espèces chacune sont les plus représentatives, viennent ensuite les Ariidae ( 3 espèces) et les Portunidae ( 2 espèces). Ce résultat peut être expliqué par l'effort de conservation consentis pour l'amélioration de la remontée biologique au niveau de l'AMP.

Niveau de satisfaction des pêcheurs après l'immersion de récifs artificiels : l'analyse de la figure 9 indique que $55,6 \%$ des pêcheurs interrogés pensent que l'immersion de récifs artificiels n'est pas satisfaisante contrairement à $44,4 \%$ qui affirment que l'activité est assez satisfaisante.

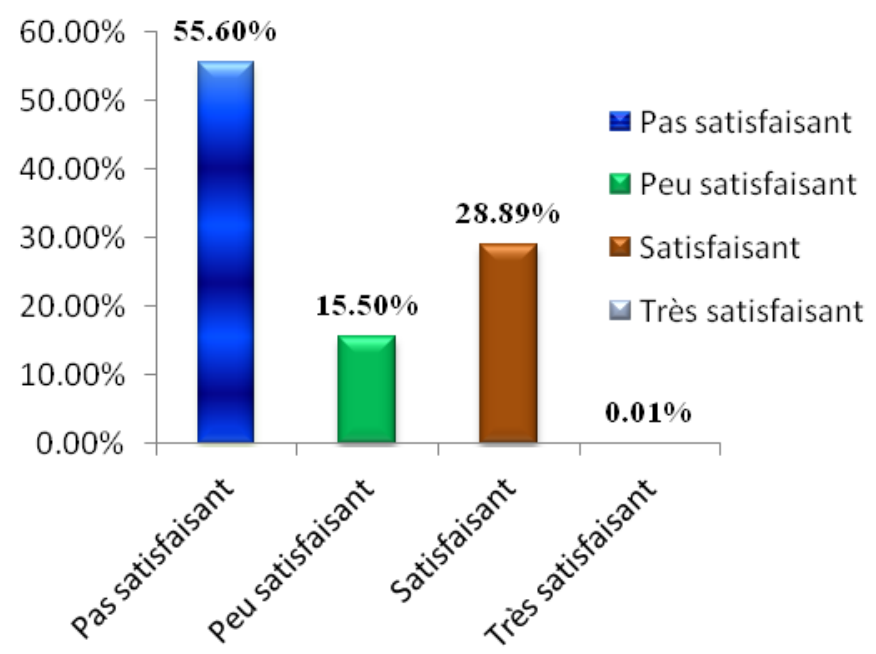

Figure 9 : Niveau de satisfaction des pêcheurs après l'immersion de récifs artificiels

- L'impact du reboisement de la mangrove sur les ressources malacologiques et sur l'avifaune: L'interrogation des femmes montre que le reboisement de la mangrove a des impacts positifs sur les ressources malacologiques à travers l'exploitation des huitres avec une production ostréicole mensuelle de $15 \mathrm{~kg}$ selon $49 \%$, 

marine et côtière (Saint-Louis, Sénégal).

$20 \mathrm{~kg}$ d'après $35 \%$ et $10 \mathrm{~kg}$ pour $16 \%$ des femmes interrogées (Figure 10). Néanmoins une baisse de la production ostréicole est notée depuis l'ouverture de la brèche, ce qui se répercute sur leur vie socioéconomique. Cependant, il est nécessaire d'intensifier le reboisement de la mangrove malgré l'impact positif noté.
Des impacts sur l'avifaune sont également notés lors de la cueillette des huîtres par la présence d'un nombre important d'oiseaux, de nids, de petits oiseaux et des œufs. Ceci confirme le rôle de lieu de refuge et de reproduction que joue la mangrove sur l'avifaune.

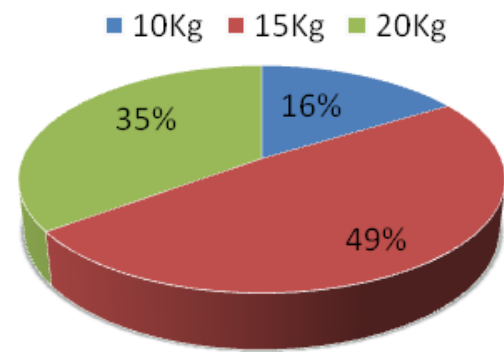

Figure 10 : Production ostréicole mensuelle des femmes interrogées

Les résultats de notre enquête effectuée au niveau des femmes transformatrices des huîtres à Diél Mbam et l'interrogation des gestionnaires ont révélé que l'activité de reboisement de la mangrove a des impacts positifs sur les ressources malacologiques et sur l'avifaune car la mangrove sert de support, de lieu de reproduction et de grossissement de plusieurs espèces halieutiques et notamment l'avifaune.
L'évaluation des ressources malacologique sur la parcelle de $90 \mathrm{~m}^{2}$ reboisée en 2012 a montré la présence de six (6) potamides (Potamide graecus) et treize (13) terriers de crabes violonistes (Uca tangeri) en moyenne dans chaque placette de $1 \mathrm{~m}^{2}$, des indices de présence d'oiseaux notamment les limicoles et des mollusques telles que les arches sont également notées dans la zone (Photo1).
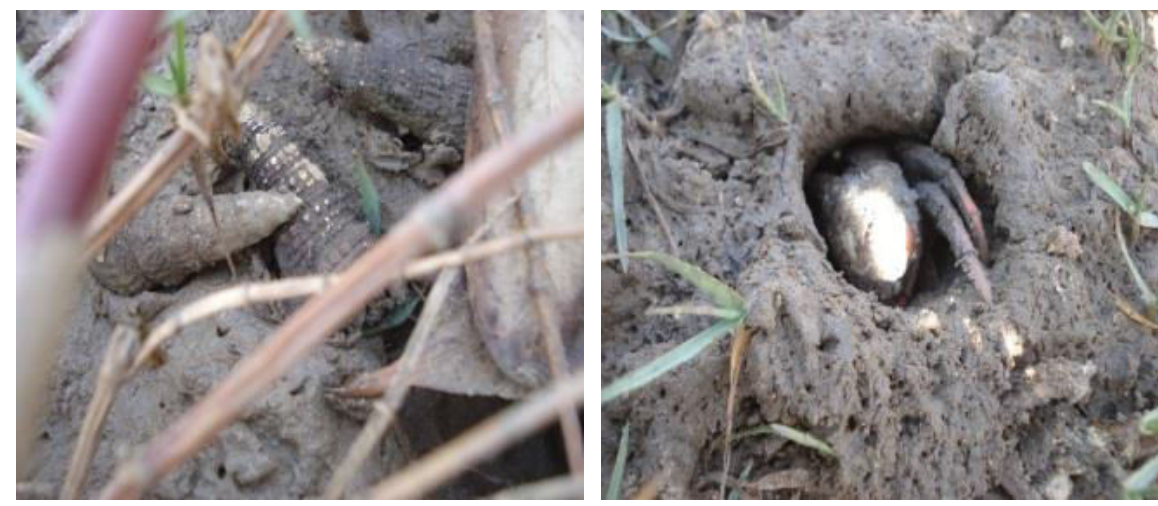

Photo 1 : Ressources malacologiques (potamides et crabe violoniste dans son terrier) retrouvées dans les placettes de mangrove (échelle : 6méga pixels)

Le processus est suivi avec la mesure des paramètres physico-chimiques environnementaux de la zone de mangrove de Diél Mbam tels que la température ambiante, la température de l'eau, le $\mathrm{pH}$, la salinité, l'humidité relative. Ces données constituent des informations supplémentaires dontl'AMP ne disposait pas (Tableau 4). 

marine et côtière (Saint-Louis, Sénégal).
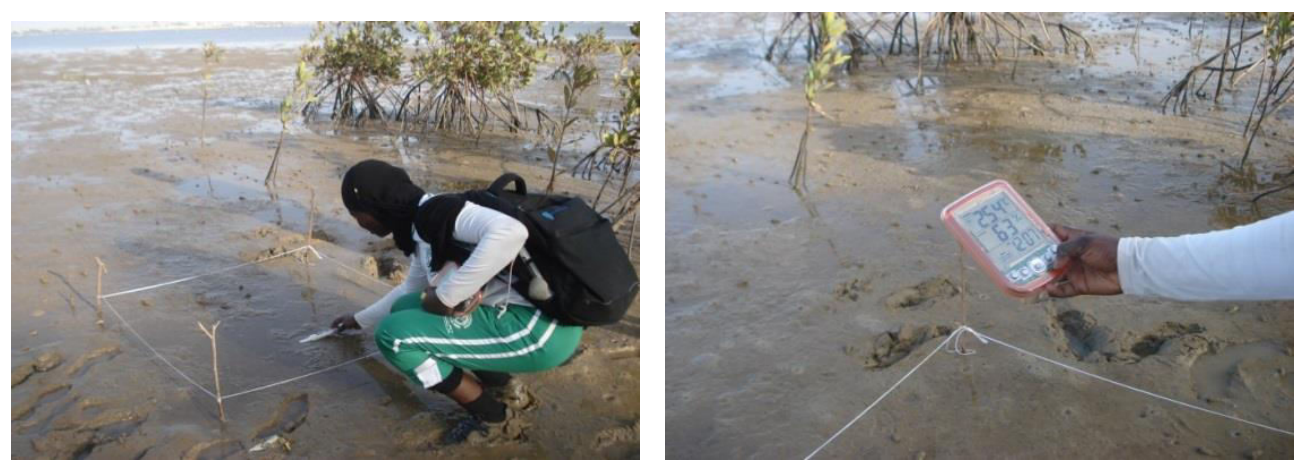

Photo 2 : Mesure des paramètres physico-chimiques de la zone de mangrove (échelle : 6 méga pixels)

Tableau 4 : Paramètres environnementaux de la zone de mangrove

\begin{tabular}{|l|l|l|l|l|}
\hline \multicolumn{5}{|c|}{ Paramètres physico-chimiques } \\
\hline $\mathrm{T}^{\circ} \mathrm{e}$ & $\mathrm{T}^{\circ} \mathrm{a}$ & $\mathrm{HR}$ & $\mathrm{pH}$ & $\mathrm{S}$ \\
\hline $25-27$ & 27,4 & 63 & $7,01-7,57$ & $22-26$ \\
\hline
\end{tabular}

$\mathrm{T}^{\circ} \mathrm{e}=$ Température de l'eau ; $\mathrm{pH}=$ Potentiel hydrogène ; $\mathrm{T}^{\circ} \mathrm{a}=$ =Température ambiante; S=Salinité ; HR=Humidité relative

\section{DISCUSSIONS}

Impacts de l'immersion des récifs artificiels: Une évolution du nombre de taxons passant de 25 à 34 ainsi que du poids des captures qui passe de $73 \mathrm{~kg}$ à 87,223 $\mathrm{kg}$ de 2009 à 2015 est enregistrée (Figure 11). Ceci est en concordance avec les résultats notés au niveau de l'AMP de Joal (Rapport d'activité, 2011) après l'immersion de récifs coquillage avec l'apparition de certaines espèces qui étaient devenues rares telles que le Mérou et des juvéniles de Tassergal au terme des quatre saisons.
Selon Claudet (2006) les récifs artificiels constituent un moyen de créer de la biomasse par l'installation de nouveaux peuplements, ces mesures de gestion concernent l'écosystème, l'ensemble des communautés. Or, les interactions entre les espèces de poissons sont complexes et les caractéristiques biologiques et les dynamiques de chaque espèce font qu'elles sont chacune affectées de manière spécifique.

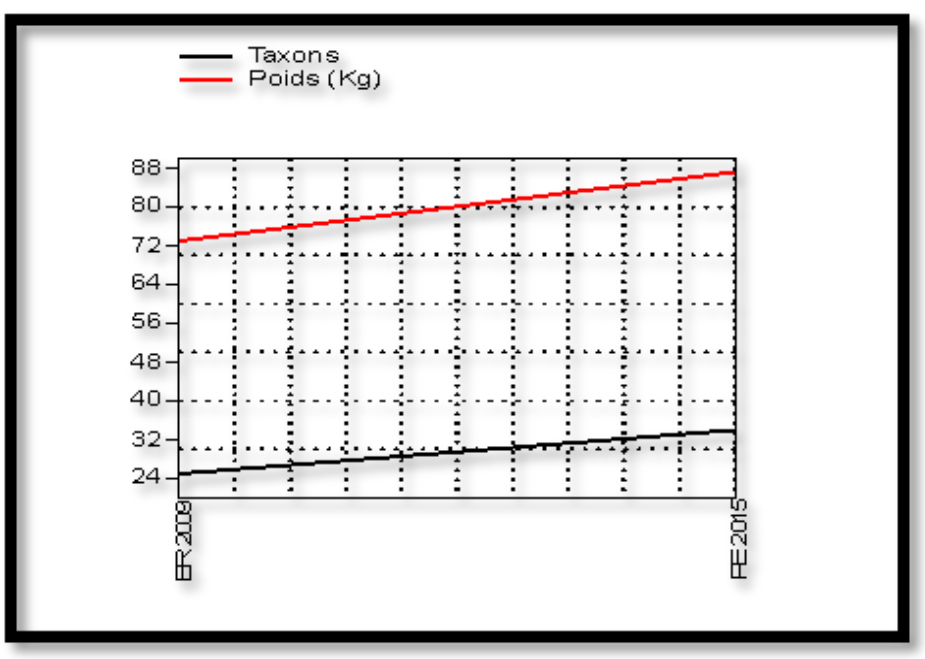

Figure 11 : Evolution des taxons et du poids des captures

Les résultats de l'enquête des pêcheurs ont révélé que l'immersion de récifs artificiels n'est pas satisfaisante et qu'aucun impact positif n'est noté selon $55,6 \%$ des interrogés contre $44,4 \%$ qui affirment que l'activité est 
assez satisfaisante car ils ont noté le retour progressif de certaines espèces indicatrices (par exemple le Mérou) et l'apparition de poulpes. En effet, les récifs constituent des habitats adéquats pour les espèces démersales. Cependant, l'évaluation de l'activité par les pêcheurs s'est basée sur une absence d'indicateurs et une configuration des récifs non adéquate à la zone immergée. Ceci est confirmé par (Claudet, 2006) qui stipule que les interactions entre la zone mise en protection et les zones adjacentes non protégées sont une source de polémiques en raison du manque de preuves permettant de décrire ces impacts et de mesurer leur importance. D'un point de vue halieutique, les AMP et les récifs artificiels ne sont pas des mesures mono-spécifiques de gestion des pêches, comme peuvent l'être les limitations en taille des individus prélevés ou des captures de certaines espèces Claudet (2006).

Impacts du reboisement de la mangrove : L'écosystème de mangrove a la particularité de pouvoir se développer dans un milieu soumis à d'énormes variations au cours du temps, rythmé par les marées et les crues sur des sols sursaturés d'eau, manquant d'oxygène et salés. La mangrove est aussi dépendante de plusieurs facteurs: la sécheresse, la gestion des grands barrages et aménagements sur le fleuve Sénégal et la dynamique urbaine. Le reboisement de la mangrove est une activité primordiale dans la gestion des zones côtières. Les résultats de l'expérience sur la parcelle de mangrove et l'interrogation des femmes transformatrices et des gestionnaires prouvent qu'elle constitue un écosystème dont le reboisement a un impact positif sur les ressources malacologiques et sur l'avifaune. Ces résultats sont en conformité avec ceux de Badji (2012) qui précise que beaucoup d'espèces vivent dans les côtes, et les critères et leur mode de vie permettent de distinguer plusieurs groupes. Ces derniers sont constitués par la faune terrestre qui comprend les oiseaux, les insectes, les reptiles et les mammifères, et la faune marine qui regroupe les huitres, les littorines et les chtamales. Les oiseaux d'eau qui s'y trouvent essentiellement prélèvent leurs proies des produits des végétaux. C'est le cas des Pélicans (Pelecanus rufescens), les Cormorans (Phalocrocorase africanus) et tant d'autres. Dans les zones de mangroves, des crabes violonistes, des poissons occupent les substrats vaseux et sablo-vaseux. La mangrove est une zone très riche en matière de ressources animales et végétales. Elle a donc besoin des conditions particulières pour sa mise en place et d'une bonne protection (Badji, 2012). Marius (1985) stipule que dans les écosystèmes de mangrove, les mollusques sont représentés par les huîtres qui se fixent sur les racines échasses des Rhizophora, auxquelles sont associés quelques gastéropodes et des bivalves. L'analyse des résultats de nos différentes études nous a permis d'affirmer que le reboisement de la mangrove a contribué partiellement à la restauration des habitats dégradés en ayant des impacts positifs sur les ressources malacologiques et sur l'avifaune.

Impacts de la brèche sur l'AMP de Saint-Louis: L'ouverture de la brèche a permis l'arrivée frontale des vagues de l'océan, ce qui provoque une érosion mécanique de la langue de Barbarie et entraîne une modification de la mangrove. Les bancs de sables (en particulier l'îlot aux oiseaux du PNLB) qui servaient de nichoirs à certains oiseaux et tortues, sont en train de disparaître à cause des eaux. De même, l'AMP de SaintLouis dont l'objectif est de promouvoir le repos biologique des espèces, donc une zone à priori tranquille et peu perturbée se connecte sur la brèche devenue le passage privilégié des pêcheurs de Guet Ndar (Niang, 2012). Donc la sensibilisation des pêcheurs sur l'importance du respect de l'AMP doit être renforcée. La construction du barrage de Diama a contribué à une déstabilisation de l'écosystème du delta dans son ensemble avec des conséquences négatives sur l'ichtyofaune. Kane (2005) estime que la biodiversité des poissons est menacée dans le cadre de l'après barrage. En effet, les ouvrages du barrage constituent des barrières physiques contre la migration des poissons et entraînent une perte d'habitat du fait du rétrécissement de la zone estuarienne. Avec le barrage, le peuplement marin inféodé à l'estuaire s'arrête à Diama et n'atteint plus ses zones de reproduction situées en amont. Globalement, on assiste à une diminution importante voire à une disparition d'espèces à affinité marine ou estuarienne comme Ethmalosa fimbriata, Tilapia guineensis etc. (Niang, 2012).

Indicateurs de suivi proposés: Dans le cadre des activités d'aménagement d'une AMP, des suivis sont très utiles. Cependant, nous proposons des indicateurs de suivi de la biodiversité halieutique et de l'avifaune qui permettront une meilleure évaluation des activités de restauration des écosystèmes de l'AMP. Relativement pour le suivi des récifs artificiels et du reboisement de la mangrove, les indicateurs biologiques et socioéconomiques suivants sont définis :

Indicateurs biologiques: En ce qui concerne les indicateurs biologiques, nous avons :

- l'augmentation ou la diminution de la taille des espèces focales capturées dans la zone de protection intégrale lors des pêches expérimentales ; 

marine et côtière (Saint-Louis, Sénégal).

- l'augmentation ou la diminution du poids moyen des espèces focales capturées dans la zone de protection intégrale lors des pêches expérimentales ;

- l'augmentation ou la diminution du nombre d'alevins capturés dans la zone de protection intégrale ; - l'apparition de nouvelles espèces indicatrices ; - la richesse spécifique des captures ;

- l'augmentation ou la diminution du nombre d'oiseaux décomptés lors des suivis aviaires ;

Indicateurs socio-économiques : Comme indicateurs socio-économiques, nous avons proposés :

\section{CONCLUSION}

L'immersion des récifs artificiels est jugée non satisfaisante selon $55,6 \%$ des pêcheurs enquêtés contre $44,4 \%$ qui pensent que l'activité est assez satisfaisante. Néanmoins, on note une évolution du nombre de taxons et du poids qui passent respectivement de 25 à 34 et de $73 \mathrm{~kg}$ à $87,223 \mathrm{~kg}$ de 2009 à 2015. En ce qui concerne le reboisement de la mangrove, l'activité est satisfaisante d'après toutes les femmes transformatrices des huîtres

\section{REMERCIEMENTS}

Les auteurs remercient vivement toute l'équipe de l'Aire Marine Protégée de Saint-Louis, les membres du comité de gestion, les partenaires et les enseignants-chercheurs du département de production forestière de l'Institut

\section{REFERENCES BIBLIOGRAPHIQUES}

Agence Nationale de Statistique et de la Démographie, 2015. Pêche à Saint-Louis, Situation économique et sociale régionale 2013, Sénégal. 87-92p, 158pages.

Badji MH, 2012. Mémoire Master II, Réhabilitation et conservation de la mangrove dans la commune de Thionck Essyl, Sénégal. 88pages.

Centre de Recherche Océanographique Dakar/Thiaroye, 2000. Pêche maritime du Sénégal. Sénégal.14pages.

Claudet J, 2006. Thèse : "Aires marines protégées et récifs artificiels : méthodes d'évaluation, protocoles expérimentaux et indicateurs". UFR de Biologie, environnement et sciences pour l'ingénieur, Université de Perpignan : 266 pages.

DIOP 0, 2006. L'exploitation agricole face aux besoins de la famille paysanne dans la région de Kaolack au Sénégal Mémoire de fin d'études ENSA . 81 pages.

Dudley N, Hockings M, Stolton S, 2000. Evaluating effectiveness: a framework for assessing the
- l'augmentation ou la baisse de revenu des pêcheurs ;

○ l'augmentation de l'effort de pêche ;

- l'augmentation de la fréquentation de la zone de pêche artisanale modérée.

Ces indicateurs déduits de l'interrogation des gestionnaires, des pêcheurs et de la recherche bibliographique, constituent des outils qui permettront de donner une information simple, quantitative et qualitative et d'évaluer la communication sur les activités d'aménagement de l'AMP de Saint-Louis.

interrogées et les gestionnaires de l'AMP. Ces activités de restauration ont des impacts relativement positifs. Cette étude sur les activités de restauration des écosystèmes dégradés de l'AMP de Saint-Louis nous a permis de montrer qu'au-delà des conséquences de la dégradation de ces écosystèmes, le potentiel de la biodiversité marine et côtière de la zone reste encore relativement important.

Supérieur de Formation Agricole et Rural (ISFAR) ex ENCR de Bambey pour leurs contributions dans nos travaux de recherches.

management of protected areas, Switzerland. 133pages.

Kane F, 2005. Evolution récente de la Langue de Barbarie (Saint-Louis du Sénégal) et ses impacts environnementaux et socioéconomiques. Mémoire de maîtrise de géographie: Université de Paris Sorbonne (Paris IV), UFR de géographie.183 pages.

Marius C, 1985. Mangroves du Sénégal et de la Gambie. Ecologie, pédologie, géochimie, mis en valeur et aménagement. France. p. 15-35. 335pages.

Niang O, 2010. Conservation de la biodiversité et développement local : l'Aire Marine Protégée de Saint-Louis. Mémoire de Master II, Université Gaston Berger de Saint-Louis, Sénégal. 94pages.

Niang O, 2012. Biodiversité halieutique et aviaire de l'Aire Marine Protégée et Saint-Louis (Sénégal): Inventaire, menaces et indicateurs de suivi. Mémoire Master II, UIPA, UCAD.68pages. 

marine et côtière (Saint-Louis, Sénégal).

Rapport d'activité, 2011. Aire Marine Protégée de Joal. Récifs coquillages. 11 pages.

WEBOGRAPHIE Commission Européenne, 2015. Pêche illicite (INN), Règles de l'UE destinées à lutter contre la pêche illicite non déclarée et non réglementée. France.

http://ec.europa.eu/fisheries/cfp/illegal fishing/in dex_fr.htm (consulté, le 18 février 2016). 